\title{
Quiescence in rice submergence tolerance: an evolutionary hypothesis
}

\author{
Chiara Pucciariello and Pierdomenico Perata
}

PlantLab, Institute of Life Sciences, Scuola Superiore Sant'Anna, Piazza Martiri della Libertà 33, 56127 Pisa, Italy

\begin{abstract}
Rice (Oryza sativa) varieties differ considerably in their tolerance to submergence, a trait that has been associated with the SUB1A gene. Recently, this gene was found in some wild rice species and landraces, which along with $O$. sativa, belong to the AA genome type group. On the basis of geographical and historical data, we hypothesize that SUB1A-1 from wild species may have been introgressed into domesticated rice. This introgression probably occurred in the Ganges Basin, with the subsequent spread of the SUB1A-1 to other areas of South Asia due to human migration. The lack of the SUB1A gene in diploid CC genome type wild rice showing submergence-tolerant traits suggests the presence of a different survival mechanism in this genetic group.
\end{abstract}

\section{Evolution of submergence tolerance in rice}

Various hydrological regimes characterize natural and anthropic habitats where flood events vary in extent, depth, frequency, and seasonal incidence. Extreme hydrological regimes include river floodplains, coastal marshes, the Amazon floodplain, as well as the areas where rice is cultivated, which are characterized by submergence of the plants that can reach several meters but can also be absent [1]. Flood-prone environments determine the distribution and abundance of plant species and exert an evolutionary pressure which selects traits that are advantageous under these environmental conditions [1]. The adaptability of a plant to waterlogging or submergence is enhanced by the development of either metabolic or anatomical characteristics [1]. These traits act in synergy to promote plant tolerance to water submergence and represent a source of genetic variations for crop breeding [2].

Rice is a unique crop due to its adaptability to different flooding conditions. The number of varieties, landraces, and wild relatives covering the large area of production is synonymous with an exceptional plasticity to diverse hydrological regimes. One of the many ways that have enabled rice to adapt to flooding has recently been identified as a 'quiescence strategy' [3]. It is characterized by reduced plant elongation during submergence, associated with regrowth when the water recedes. The ethylene responsive factor (ERF) SUBMERGENCE1A (SUB1A), in its allelic form $S U B 1 A-1$, is the key determinant of this survival mechanism [4]. SUB1A-1 is induced by ethylene, a gaseous

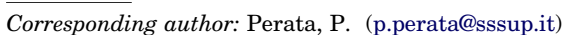

Keywords: flooding tolerance; Oryza spp.; SUB1A gene; wild rice.

$1360-1385 / \$$ - see front matter

(C) 2013Elsevier Ltd. All rights reserved. http://dx.doi.org/10.1016/j.tplants.2013.04.007 plant hormone that becomes entrapped by water submergence [5]. SUB1A-1 positively regulates the transcription of genes related to the fermentative metabolism, which is required under low oxygen conditions to sustain energy production [6]. $S U B 1 A-1$ also represses genes related to the breakdown of carbohydrates, in order to preserve the sugars needed to fuel regrowth when the water recedes [6]. Remarkably, SUB1A-1 also restricts shoot elongation, thus preventing the excessive use of carbohydrates to sustain growth, which would in any case be insufficient for the plant to reach the water surface [5]. Limited shoot elongation under submergence is achieved through the positive action of $S U B 1 A-1$ on the gibberellin (GA) signaling repressors SLENDER RICE-1 (SLR1) and SLR1-LIKE (SLRL1) [7]. During the post-hypoxic phase, SUB1A-1 is involved in acclimation to dehydration, paradoxically occurring as natural progression upon desubmergence, by increasing ABA responsiveness and reactive oxygen species (ROS) detoxification [8]. Some submergence tolerance can also be conferred by the intolerant SUB1A-2 allele, when highly expressed [9]. This allele differs in terms of the absence of the SUB1A-1 MITOGEN-ACTIVATED PROTEIN KINASE (MAPK) site [4], which might affect the activation of the protein and the regulation of downstream genes. The introgression of SUB1A-1 in rice varieties with high yields and grain quality traits, but reduced tolerance to submergence, resulted in the production of new varieties with both a high yield and submergence tolerance [10].

Considerable efforts have been made to characterize the presence/absence of the SUB1A gene in Oryza spp. [4,9,1113]. We summarize these findings on the basis of geographical origin and description of the original habitat of the genotypes studied, in order to examine the evolution of SUB1A-1 and thus the submergence tolerance trait.

SUB1A gene is present in wild and cultivated rice The genus Oryza L. consists of approximately 23 species harboring several distinct genome groups [14]. The Oryza spp. that belongs to the AA genome type includes Oryza sativa and Oryza glaberrima cultivated rice and wild species (i.e., Oryza rufipogon, Oryza nivara, Oryza barthii, Oryza longistaminata, Oryza meridionalis, and Oryza glumaepatula) [15], which can exchange genetic information when sympatric and flowering at the same time [16]. After being originally associated with the $O$. sativa small subgroup of indica varieties [4], including the aus landrace Flooding Resistant 13A (FR13A), SUB1A alleles have also been described in other genotypes including some wild 
species (e.g., O. nivara and $O$. rufipogon) $[9,12,13]$ which, together with $O$. sativa, all belong to the AA type genome group. Of the wild species belonging to the AA genomic group, $O$. rufipogon and $O$. nivara are considered to be the most likely ancestors from which $O$. sativa was domesticated [17].

Figure 1 shows a map of wild and landrace rice accessions belonging to the AA genome type investigated to date for the presence/absence of $S U B 1 A$ and with the International Rice Germplasm Collection (IRGC) number of the International Rice Research Institute (IRRI). The map shows the presence of various wild rice species harboring the SUB1A-1 allele around the basins of the Ganges and Brahmaputra rivers, which include areas of North India, Nepal, and Bangladesh. One was originally reported to be a hybrid swarm, thus probably a natural population of wild and cultivated rice interspecific hybrids. Most archeological evidence indicates the Yangtze Valley in China as the original area of rice cultivation by humans [18-21]. In addition, genetic evidence suggests that the Ganges Valley in India was a second site of potential rice domestication [22]. A phylogeographic study also showed that the southern Himalayas was the domestication region of the indica group from the $O$. rufipogon progenitor [23]. Migrants could have disseminated the seeds of submergence-tolerant plants from this area to other parts of Asia, as shown by the presence of landraces with the SUB1A-1 gene in Sri Lanka, Vietnam, and Thailand (Figure 1).

Interestingly, the presence of the intolerant allele SUB1A-2 has also been identified in $O$. sativa genotypes from China, Cuba, Nigeria, and Guinea-Bissau. The only accession of the African cultivated rice $O$. glaberrima investigated shows the absence of SUB1A and was collected in Senegal [13]. To date, only a few AA rice accessions from Africa have been analyzed, making it difficult to formulate a hypothesis as to how SUB1A introgressed in this area.

Previous surveys of AA rice genotypes revealed that the SUB1A gene was sometimes, but not always, present in $O$. nivara, O. rufipogon, and $O$. sativa [9,11-13]. This supports the theory that submergence tolerance was introgressed from divergent populations of wild relatives into cultivated $O$. sativa [13]. Wild rice species belonging to the AA genome group evolved in areas characterized by different water regimes, with $O$. nivara occurring in seasonally dry habitats, and $O$. rufipogon in permanently wet ones [14]. However, in some places $O$. nivara and $O$. rufipogon, which are cross-compatible, are sympatric in areas of rice cultivation. Despite their ecological distinction, $O$. nivara and $O$. rufipogon have similar morphological characteristics and a relatively low genetic differentiation, thus $O$. nivara is also considered as a subspecies or an ecotype of $O$. rufipogon [14,24]. One hypothesis is that $O$. nivara evolved from an $O$. rufipogon ancestor after a habitat shift [17]. The transfer of the SUB1A gene may thus have been successful in environments where flash flooding occurs at the rice seedling stage [25].

Gene flows from AA genome type wild rice species toward nearby $O$. sativa could have occurred with the introgression of key domestication alleles [26]. However, gene flows from cultivated rice could also have contributed to the genetic diversity of wild relatives in sympatric regions [21]. Studies on the genetic variation of O. rufipogon have shown a higher genetic diversity of the wild rice populations growing adjacent to cultivated rice fields than those found some distance away, thus indicating that gene flows occur from cultivated to wild rice $[27,28]$. The absence of wild rice harboring SUB1A in Sri Lanka, where SUB1Acontaining $O$. sativa landraces are present, suggests that either they have only been recently introduced in Sri Lanka or that $S U B 1 A$ was introgressed from wild rice species to $O$. sativa during domestication in the Indian Basin, and then transferred to Sri Lanka.

The introgression of $S U B 1 A$ in cultivated rice could have been a relatively recent event. Together with SUB1A (whose presence in rice cultivars is variable), other genes encoding ERFs are invariably present in the $S U B 1$ region, namely $S U B 1 B$ and $S U B 1 C$ [6]. Phylogenetic analysis has provided evidence that $S U B 1 A$ and $S U B 1 B$ diverged from $S U B 1 C$ during the differentiation between the AA and CC genome complexes [13], and that $S U B 1 A$ resulted from the duplication of $S U B 1 B$ [11]. The recent evolution of $S U B 1 A$ is also suggested by the occurrence of small allelic variations in this gene (SUB1A-1 and SUB1A-2 only) compared with the highly polymorphic $S U B 1 B$ and $S U B 1 C$ (nine and seven alleles, respectively) [4].

\section{SUB1A is absent in submergence-tolerant wild rice belonging to the $\mathrm{CC}$ genome group}

To date, the SUB1A gene has not been found in diploid wild relatives belonging to the CC type genome group Oryza officinalis, Oryza rhizomatis, and Oryza eichingeri which, however, have been shown to survive flooding by reducing growth under submergence [13]. Only SUB1C-like genes have been found in these genotypes, suggesting that this ERF may be more primitive than other SUB1 genes [13].

Wild rice belonging to the diploid $\mathrm{CC}$ genome type and not harboring the SUB1A gene have shown a disjointed distribution in Africa and Asia (Figure 2). A recent hypothesis suggested that $O$. eichingeri from Africa may have been introduced to Sri Lanka a very long time ago, because the accessions from the two locations are highly genetically divergent [24]. This species shows morphological and genetic variations in relation to geographical derivation and habitat, thus exhibiting a high degree of intraspecific variation [14]. In South Asia, wild CC and AA genotypes have been found in the same geographical region [24]. Many of the CC genotypes investigated to date have been collected in Sri Lanka where O. sativa landraces harboring SUB1A are also present (Figures 1 and 2). However, trials of breeding programs with $O$. eichingeri have reported a very low level of success in crosses with $O$. sativa [15]. Moreover, in Sri Lanka the habitat of O. rhizomatis is confined to seasonally dry zones [24], making the genetic exchange between $\mathrm{AA}$ and $\mathrm{CC}$ rice relatively difficult.

One genetic analysis determined that the $S U B 1 C$ allele, which is present in $O$. officinalis, O. rhizomatis, and O. eichingeri, does not contribute to submergence tolerance in rice [29]. Moreover, the heterologous ectopic expression of rice $S U B 1 C$ in Arabidopsis thaliana showed a limited contribution of this gene to the submergence stress response [30]. Overall, this supports the idea of a molecular 


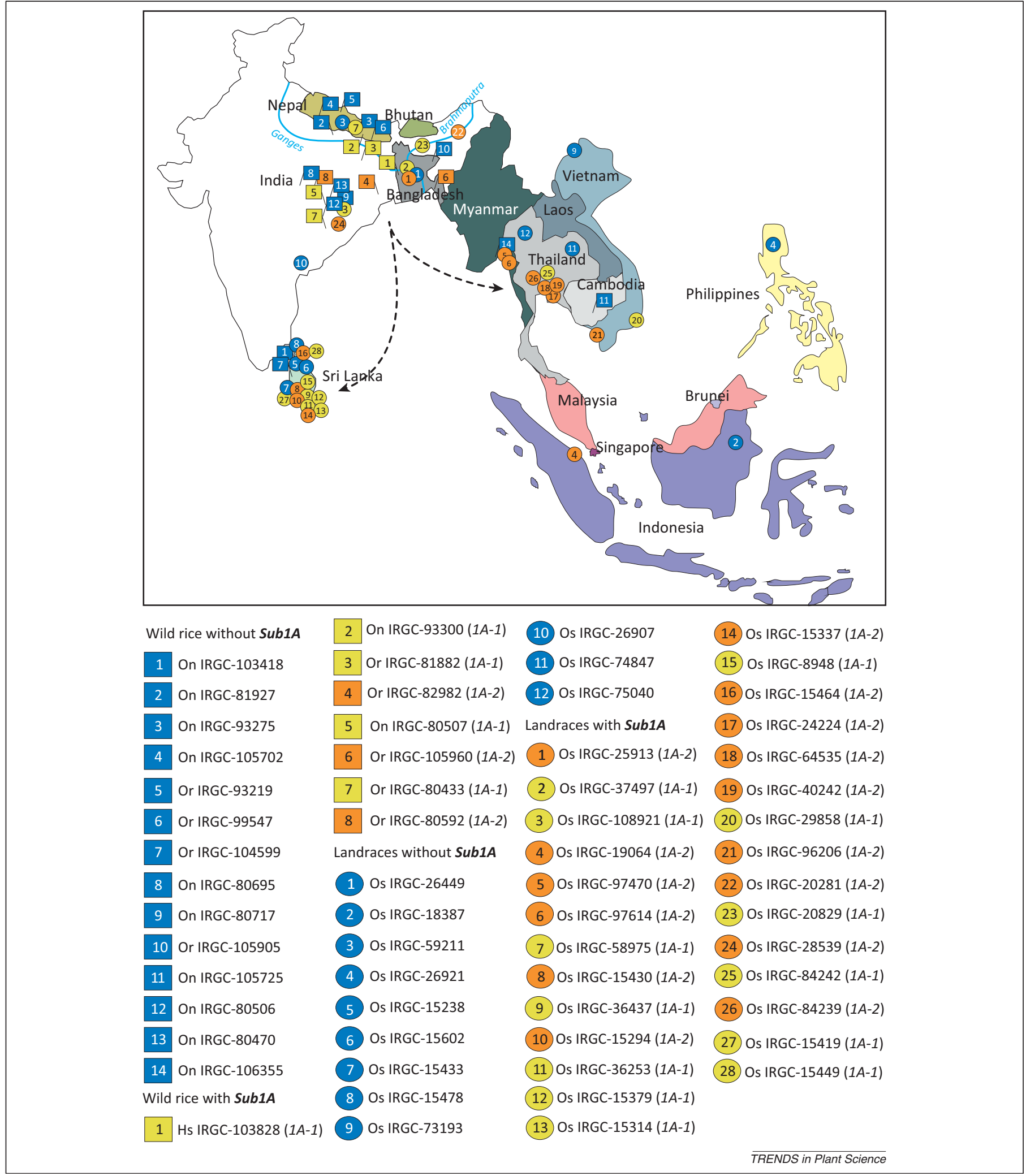

Figure 1. Location of the original collection sites of AA genome type wild genotypes (square symbols) and landraces (circle symbols), with or without SUB1A genes [9,1113]. Wild genotypes and landraces without either SUB1A-1 or SUB1A-2 are represented by blue symbols. Genotypes with the tolerant allele SUB1A-1 are indicated as ' $1 A-1$ ' in the key and yellow squares or circle symbols in the map, while those harboring the intolerant allele $S U B 1 A-2$ are indicated with ' $1 A-2$ ' in the key and orange squares or circles in the map. The accessions shown represent those investigated to date for the presence/absence of SUB1A and with the International Rice Germplasm Collection (IRGC) number of the International Rice Research Institute (IRRI). The source country and collection site of the AA genome type wild rice accessions were studied using the original collection forms of the International Rice Information System (IRIS) from the IRRI (http://www.irri.org), whereas landraces were investigated with information obtained by the System-wide Information Network for Genetic Resources (SINGER), now part of the Plant Genetic Resource Gateway (GENESYS, http://www.genesys-

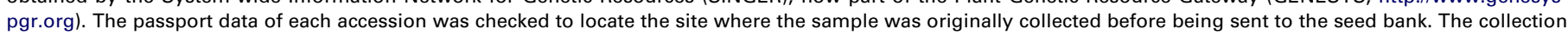
site included a wild zone or a field border for the wild species and the germplasm location before being sent to the gene bank for landraces. Rice species with unspecified or other statuses (e.g., improved cultivar and crosses) were not included in the analysis, and neither were the wild accessions lacking the original IRRI collection form. The dotted arrows represent the directions in which domesticated rice may have spread. 


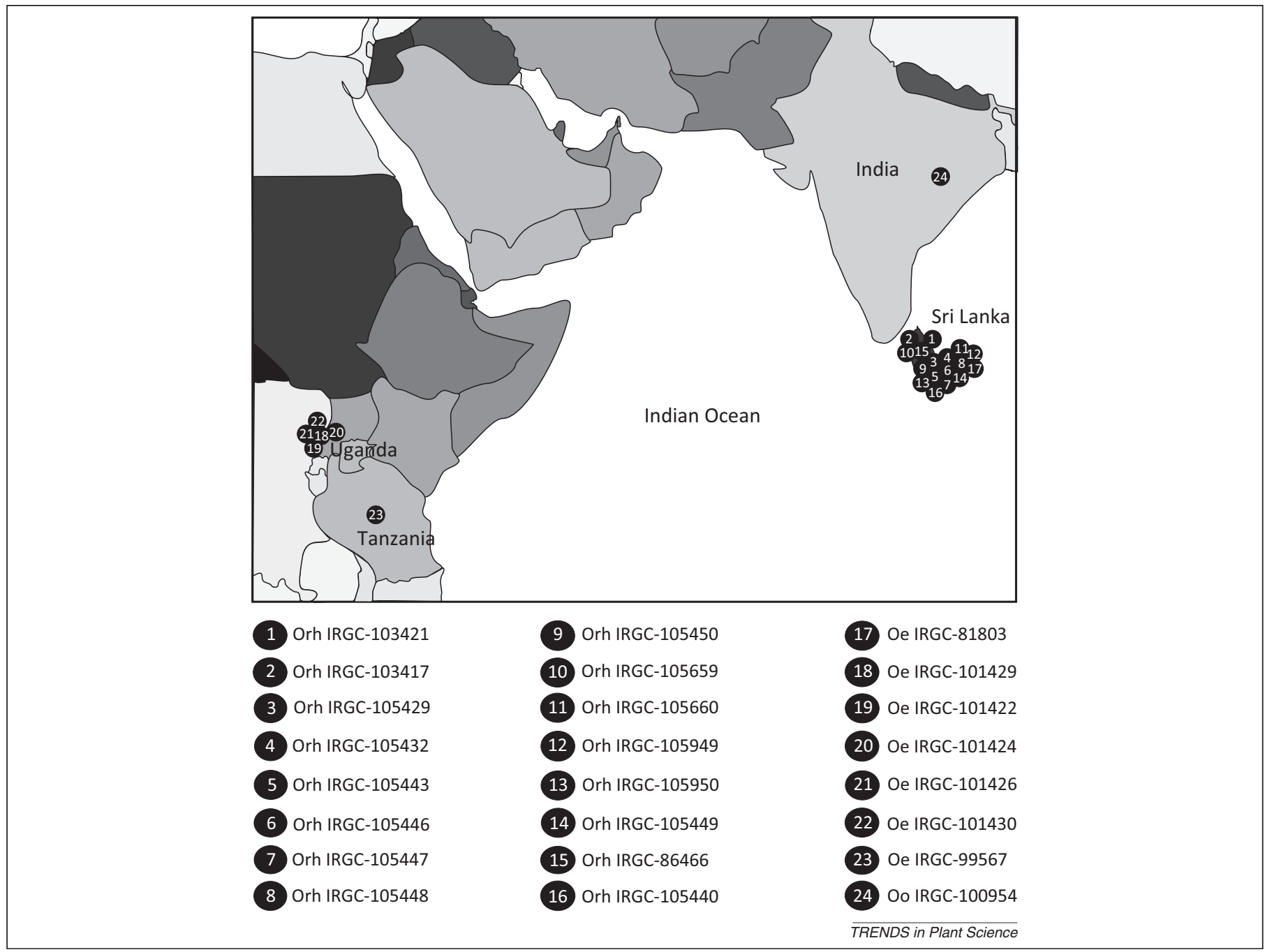

Figure 2. Location of the original collection sites of diploid CC genome type wild genotypes not harboring the SUB1A gene [13]. The source country and collection site of the CC genome type wild rice accessions were studied using the original collection forms of the International Rice Information System (IRIS) from the International Rice Research Institute (IRRI) (http://www.irri.org). For some of these accessions, even if the country source was indicated, the typology of the original collection site was unknown.

mechanism of submergence tolerance which may be unique to the CC type genome group and which still needs to be found. The rice varieties Madabaru and IR72 have recently been shown to harbor novel submergence tolerance quantitative trait loci (QTLs), which are independent of the ethylene-mediated SUB1A pathway [31].

\section{Concluding remarks}

Various studies have highlighted that the presence of SUB1A alleles is highly variable in several wild and landrace accessions of rice belonging to the AA genome group. Analysis of the geographical distribution of wild rice and landrace data, together with the molecular analysis for the presence of $S U B 1$ genes, suggests that the introgression of $S U B 1 A-1$ from wild into cultivated rice originated around the Ganges Basin. The presence of SUB1A-1 in O. sativa landraces present in other Asian regions suggests that early settlers may have brought with them the seeds of submergence-tolerant plants.

Submergence-tolerant traits have also been shown to be present in some diploid CC type wild rice accessions which, however, lack SUB1A. Only $S U B 1 C$-like genes have been found in these genotypes; therefore, it is possible that they harbor a distinctive molecular mechanism of submergence tolerance, which is still unknown. In South Asia, these genotypes are found in the same geographic region where AA rice species grow; however, success in intergenomic crosses is very low.

Novel QTLs associated with submergence-tolerant traits might be useful in enhancing the level of survival. Indeed, the hybridization of wild and cultivated rice with the introgression of alien genes can give rise to new interesting variations, due to the high level of genetic diversity. Rice breeding programs that could overcome the problem of cross-compatibility barriers would be a breakthrough in rice enhancement.

\section{Acknowledgments}

We would like to thank Julia Bailey-Serres for helpful discussions, Claudio Pugliesi for reading the manuscript, and Sandro Parlanti for his assistance in preparing the manuscript.

\section{References}

1 Colmer, T. and Voesenek, L.A.C.J. (2009) Flooding tolerance: suites of plant traits in variable environments. Funct. Plant Biol. 36, 665-681

2 Bailey-Serres, J. et al. (2010) Submergence tolerant rice: SUB1's journey from landraces to modern cultivar. Rice 3, 138-147 
3 Bailey-Serres, J. and Voesenek, L.A.C.J. (2008) Flooding stress: acclimations and genetic diversity. Annu. Rev. Plant Biol. 59, 313-339

$4 \mathrm{Xu}, \mathrm{K}$. et al. (2006) Sub1A is an ethylene-response-factor-like gene that confers submergence tolerance to rice. Nature 442, 705-708

5 Perata, P. and Voesenek, L.A.C.J. (2007) Submergence tolerance in rice requires $S u b 1 A$, an ethylene-response-factor-like gene. Trends Plant Sci. $12,43-46$

6 Fukao, T. et al. (2006) A variable cluster of ethylene responsive-like factors regulates metabolic and developmental acclimation responses to submergence in rice. Plant Cell 18, 2021-2034

7 Fukao, T. and Bailey-Serres, J. (2008) Submergence tolerance conferred by Sub1A is mediated by SLR1 and SLRL1 restriction of gibberellin responses in rice. Proc. Natl. Acad. Sci. U.S.A. 105, 16814-16819

8 Fukao, T. et al. (2011) The submergence tolerance regulator SUB1A mediates crosstalk between submergence and drought tolerance in rice. Plant Cell 23, 412-427

9 Singh, N. et al. (2010) Molecular marker survey and expression analyses of the rice submergence-tolerance gene SUB1A. Theor. Appl. Genet. 121, 1441-1453

10 Singh, N. et al. (2009) Responses of SUB1 rice introgression lines to submergence in the field: yield and grain quality. Field Crop Res. 113, $12-23$

11 Fukao, T. et al. (2009) Evolutionary analysis of the Sub1 gene cluster that confers submergence tolerance to domesticated rice. Ann. Bot. 103, $143-150$

$12 \mathrm{Li}, \mathrm{Z}-\mathrm{X}$. et al. (2011) Identification of SUB1A alleles from wild rice Oryza rufipogon Griff. Genet. Resour. Crop Evol. 58, 1237-1242

13 Niroula, R.K. et al. (2012) SUB1A-dependent and -independent mechanisms are involved in the flooding tolerance of wild rice species. Plant J. 72, 282-293

14 Vaughan, D.A. and Morishima, H. (2003) Biosystematics of the genus Oryza. In Rice: Origin, History, Technology and Production (Wayne Smith, C. and Dilday, R.H., eds), pp. 27-65, John Wiley and Sons

15 Vaughan, D.A. et al. (2003) Diversity in the Oryza genus. Curr. Opin. Plant Biol. 6, 139-146

16 Doi, K. et al. (2008) Genetic variation in rice. Curr. Opin. Plant Biol. 11, $144-148$

17 Sang, T. and Ge, S. (2007) Genetics and phylogenetics of rice domestication. Curr. Opin. Genet. Dev. 17, 533-538
18 Normile, D. (1997) Archaeology: Yangtze seen as earliest rice site. Science 275, 309-310

19 Jiang, L. and Liu, L. (2006) New evidence for origins of sedentism and rice domestication in the lower Yangzi River, China. Antiquity 80, $355-361$

20 Zheng, Y.F. et al. (2007) Characteristics of the short rachillae of rice from archaeological sites dating to 7000 years ago. Chin. Sci. Bull. 52, $1654-1660$

21 Vaughan, D.A. et al. (2008) The evolving story of rice evolution. Plant Sci. 174, 394-408

22 Fuller, D. (2003) An agricultural perspective on Dravidian historical linguistics: archaeological crop packages, livestock and Dravidian crop vocabulary. In Examining the Farming / language Dispersal Hypothesis (Bellwood, P. and Renfrew, C., eds), pp. 191-213, McDonald Institute for Archaeological Research

23 Londo, J.P. et al. (2006) Phylogeography of Asian wild rice, Oryza rufipogon reveals multiple independent domestications of cultivated rice, Oryza sativa. Proc. Natl. Acad. Sci. U.S.A. 103, 9578-9583

24 Bautista, N. et al. (2006) Genetic diversity in AA and CC genome Oryza species in southern South Asia. Genet. Resour. Crop Evol. 53, 631-640

25 Nagai, K. et al. (2010) Stunt or elongate? Two opposite strategies by which rice adapts to floods. J. Plant Res. 123, 303-309

26 Kovach, M.J. et al. (2007) New insights into the history of rice domestication. Trends Genet. 23, 578-587

27 Song, Z.P. et al. (2003) Gene flow from cultivated rice to the wild species Oryza rufipogon under experimental field conditions. New Phytol. 157, 657-665

28 Cai, H-W. et al. (2004) Comparison of population genetic structures of common wild rice (Oryza rufipogon Griff.), as revealed by analyses of quantitative traits, allozymes, and RFLPs. Heredity 92, 409-417

29 Septiningsih, E.M. et al. (2009) Development of submergence-tolerant rice cultivars: the Sub1 locus and beyond. Ann. Bot. 103, 151-160

30 Peña-Castro, J.M. et al. (2011) Expression of rice SUB1A and SUB1C transcription factors in Arabidopsis uncovers flowering inhibition as a submergence tolerance mechanism. Plant J. 67, 434-446

31 Septiningsih, E.M. et al. (2012) Identifying novel QTLs for submergence tolerance in rice cultivars IR72 and Madabaru. Theor. Appl. Genet. 124, 867-874 